\title{
Bridging Between Plastic Surgery and Psychiatric Medicine: Re- directing Approach
}

\author{
Ahsan Zil E Ali ${ }^{1 *}$, Muhammad Aadil' ${ }^{2}$, Aitzaz Munir ${ }^{3}$ \\ ${ }^{1}$ Deparrtment of Surgery, Fatima Memorial Hospital, Lahore, Pakistan \\ ${ }^{2}$ Department of Psychiatry, Rush University Medical Center, US \\ ${ }^{3}$ Departmentof Psychiatry, Howard University Hospital, Maryland, US
}

"Corresponding author: Ahsan Zil E Ali, Department of Surgery, Fatima Memorial Hospital, Lahore, 725 Shadman Rd, Lahore 54000, Pakistan. Tel: +9242111555600; Email: ahsanzileali@gmail.com

Citation: Ali AZE, Aadil M, Munir A (2017) Bridging Between Plastic Surgery and Psychiatric Medicine: Redirecting Approach. J Surg: JSUR-161. DOI: 10.29011/JSUR-161.000061

Received Date: 05 August 2017; Accepted Date: 07 August 2017; Published Date: 14 August, 2017

\section{Editorial}

Plastic surgery and its reconstructive aspect has entirely changed the way we see a human body. In past few years, it's been a popular surgical science that does not only deal with traumatic injuries but also with anti-aging, body sculpturing and transformation procedures. This rise has been up to $34 \%$ from 2005 to 2006, alone in one-year time, which shows it as a promising surgical specialty that has offered a lot to our race[1]. But despite of the popularity of these procedures, the controversial psychological outcomes and overlapping Body Dysmorphic Disorder (BDD) and unsatisfied self-image prevails. A popular news reported in 2015 stated the killing of a plastic surgeon by a patient for 'Unsatisfactory plastic procedure', apparently the patient had multiple procedures for an unsatisfactory nose shape and kept on visiting his doctor for 5 years[2]. Another homicidal threat was recorded in Facial Plastic Surgicenter of Baltimore where the patient threatened by guns in his pocket and the only reason for this act was 'Nose not quite what he wanted'. In another episode, Reid Ewing, a popular American actor stated that he has body dysmorphia and has undergone multiple procedures without psychiatric screening. Dr. Ishii, a facial plastic surgeon from Johns Hopkins School of Medicine reacted to the prevalent psychiatric diseases among these patients as, "We are not psychiatrists, we are surgeons, so it's unreasonable for us to guess whether someone has a mental illness" $[3,4]$.

These recorded events have proven that seeking multiple plastic surgery consults and procedures have a lot to do with prevalent psychiatric illnesses that range from body dysmorphia, depression and suicidal or homicidal ideation. Breast augmentation is few of the most popular cosmetic intervention but the psychiatric outcomes were alarming. Briton, et al. presented the results of their extensive 13,000 women cohort of females undergoing breast augmentation which revealed a Mortality Rate (MR) of 1.54 in
14 years and 1.63 in 5 years by suicide. Koot, et al. did a similar survey in Sweden that showed even higher MR with 2.9, whereas another recorded study among Danish and Finnish women was as high 3.1 and 4.26 MR, respectively[5,6]. These known MRs are actually a red flag to the surgical practice and the patient's care. Even the epidemiological distribution of BDD in population is itself very high with $1 \%$ and the patient's undergoing plastic surgeries is even higher between $7-15 \%[7,8]$.

Considering all these statistics, it becomes essential that a psychiatric screening and evaluation of the results can redirect a patient from seeking a surgery to a cognitive treatment and planning that can make the patient satisfied rather than having multiple procedures. Such important intervention can reduce the mortality rates and increase patient's satisfaction for him or herself. Also, there can be reduction in burden of this practice that eventually leads to multiple procedures without addressing the root psychiatric issue. There can be multiple ways to intervene once the plastic surgeon and psychiatrist is towards one direction.

A surgeon can have a preliminary past history evaluation to look for any psychiatry related event, psychiatry consults or any mood related disorders. It is also important to see for consumption of any illicit drug or medications. Once the patient's records are considered clear, a surgeon can ask the patient to respond to Body Dysmorphic Disorder Questionnaire (BDDQ) which is a 4-question item. This can be a very basic way to know the status without asking a psychiatric session. If the interpretation is likely to have this condition, the patient can be referred for a psychiatric consult which will involve extensive questioning, familial factors and environmental conditions that may have led to this disorder. At this point, the patient should not have a surgical consult or a surgeon should not plan any plastic or reconstructive procedure. Instead, a good cognitive behavioral plan should be devised by the psychia- 
Citation: Ali AZE, Aadil M, Munir A (2017) Bridging Between Plastic Surgery and Psychiatric Medicine: Redirecting Approach. J Surg: JSUR-161.

trist and periodic psychological evaluations are planned.

Bridging between plastic surgery and psychiatric medicine can be novel and very smart practice that can change this cosmetic field and can positively affect the outcomes of this practice. The results of BDDQ in plastic surgery service prior to the procedure have shown that $13.1 \%$ of patients undergoing cosmetic surgery and $1.8 \%$ of patients undergoing a reconstructive procedure had BDD, which otherwise have gone undiagnosed [9]. This can be a step-up in daily healthcare practice of a plastic surgeon and can be an innovative way of directing the patient to right treatment.

\section{References}

1. American Society of Facial Plastic and Reconstructive Surgeons 8. (AAFPRS) 2006 Membership Survey: Trends in Facial Plastic Surgery. Alexandria, VA: American Society of Facial Plastic and Reconstructive Surgeons 2006.

2. Amos H (2017) Russian unhappy with cosmetic surgery shoots doctor.
3. STAT (2017) Plastic surgeons increase patient psychiatric screenings.

4. Huff Post (2017) I Underwent Cosmetic Surgery for My Body Dysmorphia... And I Wish I Hadn't.

5. Brinton LA, Lubin JH, Burich MC, Colton T, Hoover RN (2001) Mortality among augmentation mammoplasty patients. Epidemiol 12: 321-326.

6. Koot VCM,Peeters PHM, Granath F, DE Grobbee, O Nyren (2004) Total and cause specific mortality among Swedish women with cosmetic breast implants: prospective study. Arch Int Med 164: 2450-2455.

7. Glaser DA and Kaminer MS (2005) Body dysmorphic disorder and the liposuction patient. DermatolSurg31: 559-560.

8. Sarwer DB, Wadden TA, Pertschuk MJ, Whitaker LA (1998) Body image dissatisfaction and body dysmorphic disorder in 100 cosmetic surgery patients. PlastReconstrSurg 101: 1644-1649.

9. Dey JK, Ishii M, Phillis M, Byrne PJ, Boahene KD, et al. (2015) Body dysmorphic disorder in a facial plastic and reconstructive surgery clinic: measuringprevalence, assessing comorbidities, and validating a feasible screening instrument. JAMA Facial PlastSurg 17: 137-143. 